\title{
Vicenza: una normativa a la espera que se aplique
}

Vicenza: pending legislation that applies

\author{
Claudia García Ochoa e Ignacio Corvalán Rossel
}

\section{Filiación}

Participantes en el curso para jóvenes profesionales dictado por el Centro Internacional de Formación para la Valorización y Conservación de los contextos histórico urbanos (Italia), representando a la Ilustre Municipalidad de Santiago de Chile.

nachoenitalia@hotamail.com

\section{Resumen}

Se presenta el estudio realizado en 2003 para revitalizar Vicenza, ciudad italiana cuyo particular patrimonio arquitectónico está constituído por las obras de Andrea Palladio, las que llevaron a la UNESCO a incluirla en 1994 en la lista del Patrimonio Mundial.

\section{Palabras clave}

Vicenza, Andrea Palladio, normativa y patrimonio, patrimonio mundial.

\section{Abstract}

This article presents the 2003 study to revitalize Vicenza, this city's major heritage attaction are the buildings designed by Andrea Palladio. The city was included in the world heritage list in 1994.

\section{Key words}

Vicenza, Andrea Palladio, cultural heritage conservation, world heritage.

\section{Sumario}

Introducción

Antecedentes generales de la ciudad de Vicenza

Antecedentes históricos en relación con la evolución del tejido urbano de Vicenza

Antecedentes normativos

El plano seccional del centro histórico

Problemática actual

Red de cooperación urb-al

Vicenza, el nuevo desarrollo urbano a partir del Programa de Recalificación Urbana y del Desarrollo Sostenible del Territorio, P.R.U.S.S.T

Bibliografía 


\section{Introducción}

Vicenza se encuentra en una de las siete provincias de la Región del Véneto. En la provincia de Vicenza, Comuna de Vicenza, está al noreste de la península itálica. En 1994 fue incluida en la lista de Patrimonio Mundial de la UNESCO por las obras de Andrea Palladio.

En 1963 Mario Coppa estudia revitalizar la ciudad de Vicenza. Este estudio sería la base para la generación del Plano Particularizado del Centro Histórico, pero este no generó soluciones a todos los problemas detectados. En 1969 se generan distintos proyectos, de los cuales pocos fueron llevados a cabo en los plazos planificados. Pese a la actual demanda de habitación en el centro histórico, este continúa siendo un espacio poco habitado, que terminada la jornada laboral queda prácticamente desierto.

En noviembre del 2003 se desarrolla un P.R.U.S.S.T. cuyo principal objetivo es garantizar un centro moderno, funcional, promoviendo la calidad de vida y la vigilancia del medio ambiente. Todo esto en un complejo programa de realización de 27 proyectos, este programa tiene un plazo de diez años. En esta acción se pueden dilucidar las conclusiones generales anteriormente propuestas por Coppa en 1963, que todavía esperan una solución a pesar del tiempo transcurrido.

\section{Antecedentes generales de la ciudad de Vicenza}

Vicenza corresponde a una de las siete provincias de la región del Véneto, cuya capital es la ciudad de Vicenza. Está emplazada en el norte de la península itálica, entre los Alpes Dolomitas y el mar Adriático.

Actualmente la Comuna de Vicenza posee 107.223 habitantes dentro de una superficie de $80,46 \mathrm{Km} 2$, con una densidad de 1332,62 hab/ km2.

En diciembre de 1994, Vicenza, "Ciudad de Palladio", fue incluida en la Lista de Patrimonio Mundial de la UNESCO, por contener los bienes arquitectónicos palladianos, de interés excepcional y de valor universal, toda vez que han ejercido una gran influencia sobre la cultura mundial.

\section{Antecedentes históricos en relación con la evolución del tejido urbano de Vicenza}

En el siglo II a. C. los romanos comienzan a colonizar la llanura Padana, lo que trajo consigo numerosas obras, entre las que destacan el trazado de nuevas vías como la Postumia, Emilia y Popillia Annia, y la construcción de diques que ordenaron los trazados irregulares de los ríos, mejorando los terrenos pantanosos de la llanura véneta. El recorrido de la Vía Postumia, nace como conexión entre Génova y Aquilea; en el 148 a. C., la aparición de esta vía determinó el destino de Vicenza, llevándola a ser sede de un municipio romano.

La fundación romana de la ciudad se realiza en torno al año 49 a. C. Se calcula que el área ocupada tuvo como dimensiones $650 \mathrm{~m} \times 500 \mathrm{~m}$, su trazado tomó la clásica forma reticular de isla romana, la "citta Quadrata". En la actualidad aún es posible leer el trazado fundacional romano; es así que la vía principal, llamada Corso Palladio, eje central 
longitudinal, corresponde al antiguo decumano mayor y la vía denominada Contra Porti corresponde al cardo mayor.

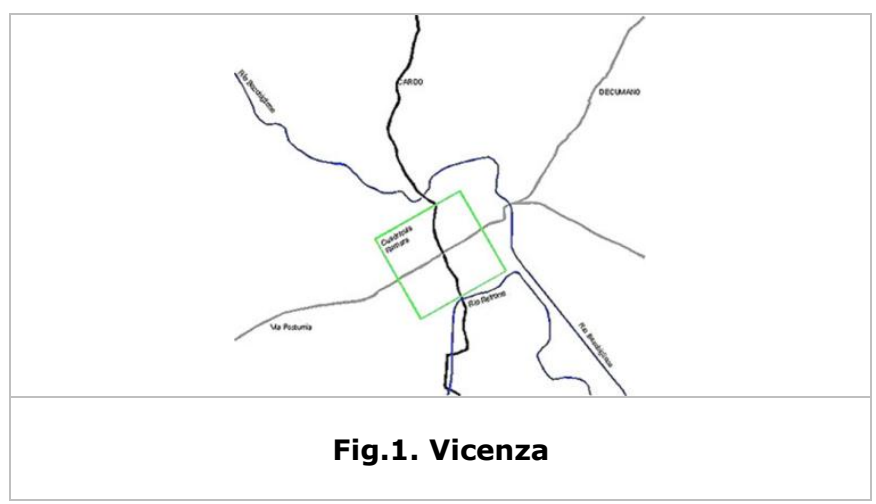

Vicenza estuvo bajo diversos dominios, así, durante el siglo VI la ciudad pasa a ser sede de uno de los 36 ducados lombardos; a fines del siglo VII pasa a ser parte del imperio carolingio. Tras la caída de este último, sigue un peligroso período de anarquía en Italia en donde muchos grupos de nobles se disputaban el poder. Es así que, en el siglo X, se comienza la construcción de la muralla defensiva.

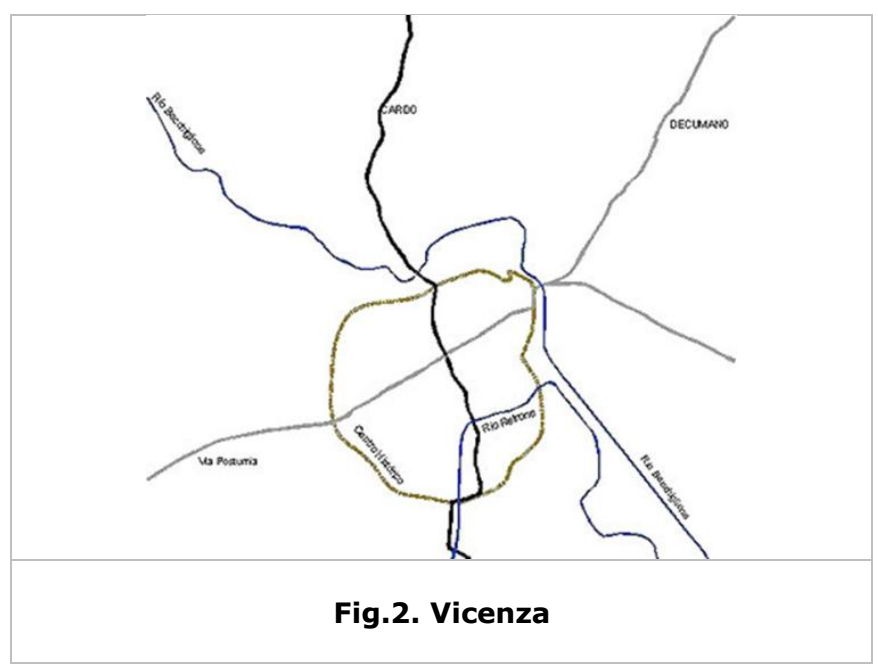

En este momento la ciudad se dilata tendiendo a asumir la forma redonda típica de muchas ciudades del alto medioevo, inspirada en Jerusalén, la ciudad santa por excelencia. Vicenza queda contenida por un muro en anillo, abierta sobre el territorio a partir de cuatro puertas que corresponden a los cuatro barrios principales y cuatro ingresos secundarios. 


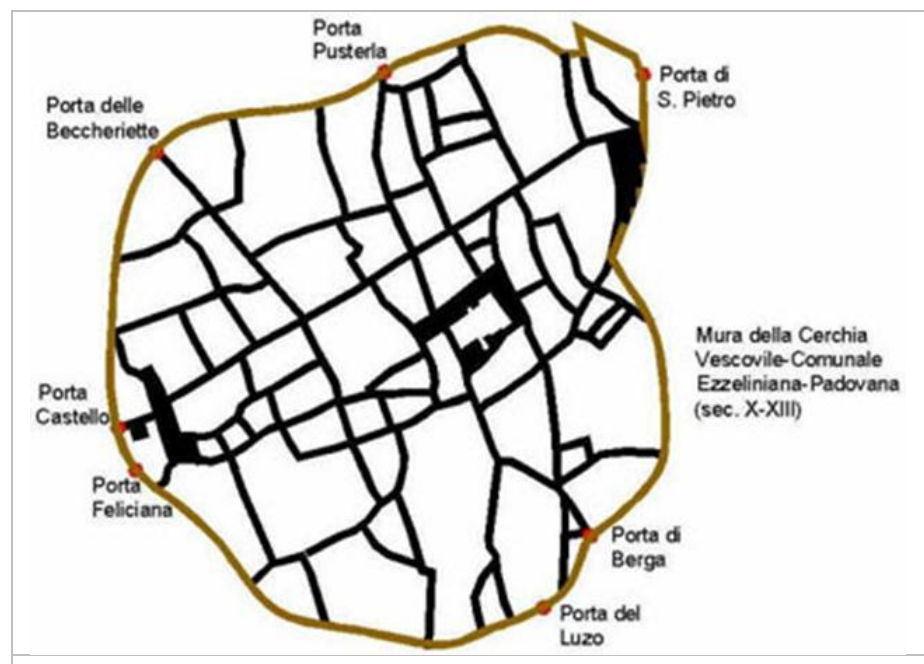

Fig.3. Vicenza. Trazado vial.

La construcción del muro continuará hasta el siglo XIII, a lo largo de los años se añadieron nuevas áreas a la ciudad denominadas adiciones, en donde se ampliaba consecuentemente el perímetro de la muralla. La primera de estas adiciones se lleva a cabo a finales del 1200, incorporando un área en el sector sudeste. Esta expansión se realiza durante un período en que la ciudad estaba custodiada por la comuna de Padua y la familia de Carrara.

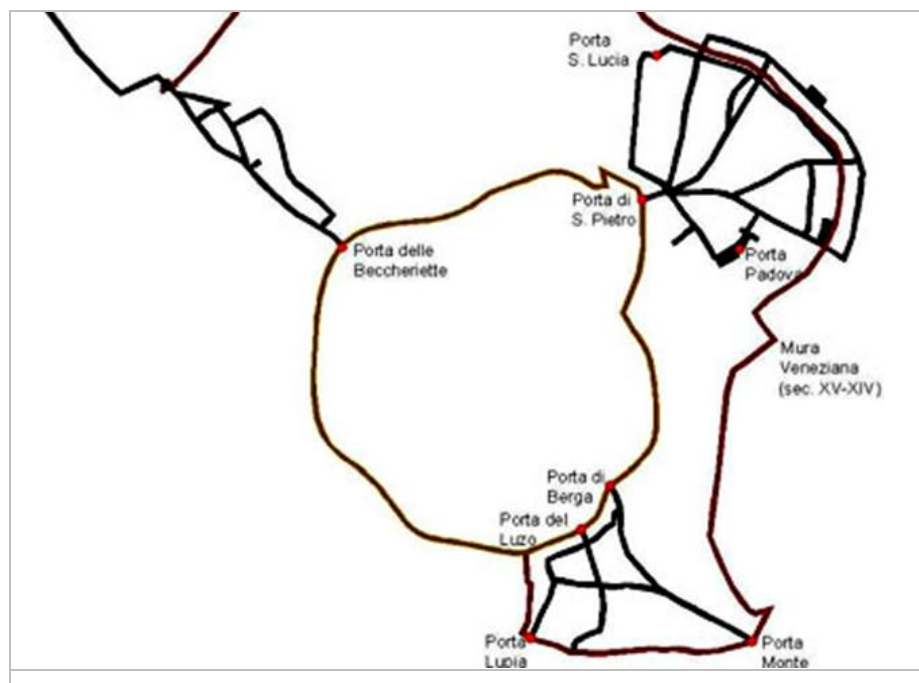

Fig.4. Vicenza

Más tarde, en 1311, Vicenza pasa a manos de una familia de Verona, los señores de la Scala; durante este dominio se realiza una importante expansión de la cinta amurallada, 
creándose el barrio de Porta Nuova el cual fue trazado a partir de una malla ortogonal que contrastaba con los trazados preexistentes.

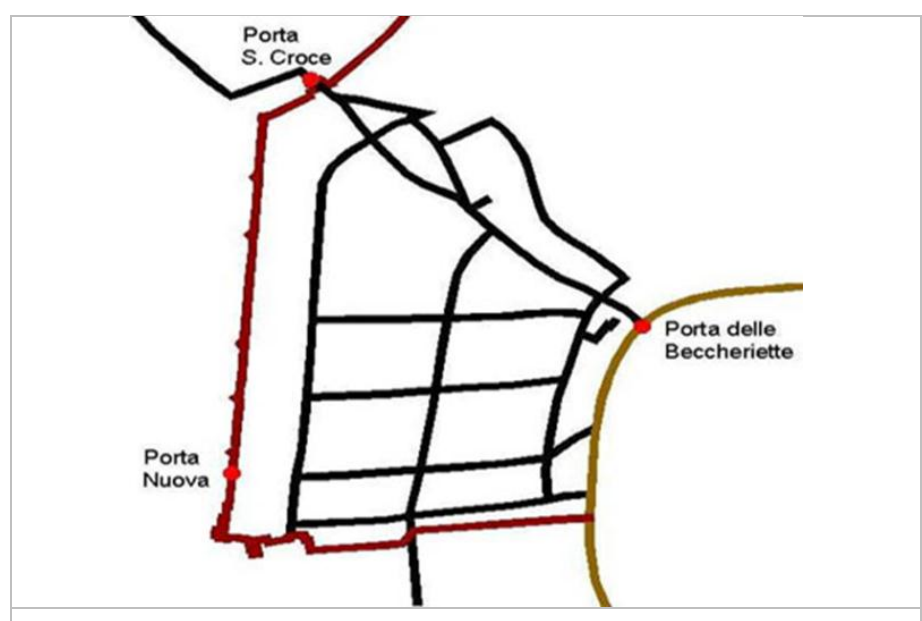

Fig.5. Vicenza

Tras la caída de estos señores de Verona, sucede un breve paréntesis en donde la ciudad estuvo en manos de los Visconti, que no dejaron mayor huella en el tejido urbano.

La conquista veneciana de Vicenza se llevó a cabo en el año 1404, y esta conquista puso fin a siglos de contrastes y guerras, inaugurando condiciones de estabilidad política, de sabiduría en la administración y de seguridad militar. A partir del siglo XVI, Venecia da vida a un extraordinario proyecto de reorganización del espacio físico y reordenamiento del sistema hidráulico de toda el área del Véneto, el cual estuvo bajo su dominio hasta el año de su caída en 1797.

Con ello se comienza a construir bajo nuevos parámetros $y$, entre los muchos aspectos modificados, está el cambio de las casas patronales y de las villas tanto nuevas como existentes, agregando un estilo y un sistema constructivo que dan nueva un nuevo rostro a las edificaciones anteriormente góticas o características del siglo XV.

La República veneciana, después de la reconquista del territorio, demolió gran parte de las instalaciones medievales, sustituyendo las torres por las logias renacentistas indefensas y abiertas al paisaje ${ }^{1}$.

Andrea di Pietro della Gondola, más conocido como Palladio, es un ejemplo clarísimo de este mensaje cultural. Vicenza y sus alrededores tuvieron el privilegio de acoger el núcleo central de la experiencia palladiana, con numerosas obras, entre las que se destacan el Pallazzo Municipale, más conocida como la Basílica, cuya columnata fue diseñada por Andrea Palladio en el año 1549; el Palacio Barbaran, el Palacio Thiene, la Villa Capra Valmarana, más conocida como "La Rotonda", el Teatro Olímpico y tantas otras obras más.

Hasta la época de la unificación italiana (1861), el núcleo de Vicenza se mantuvo prácticamente contenido en los límites de la doble cinta muraria, la ciudad estaba

1 FOSCARI, Antonio, "Torri e logge", en el libro Paisaje Veneto, 1984, Editorial Silvana. 
amurallada por una cinta de origen medieval y otra veneciana, sin embargo el área de la ciudad al exterior de los muros continuó desarrollándose en torno a las principales rutas de conexión con otros centros urbanos; estas directrices reafirmaron su diseño de ciudad radial.

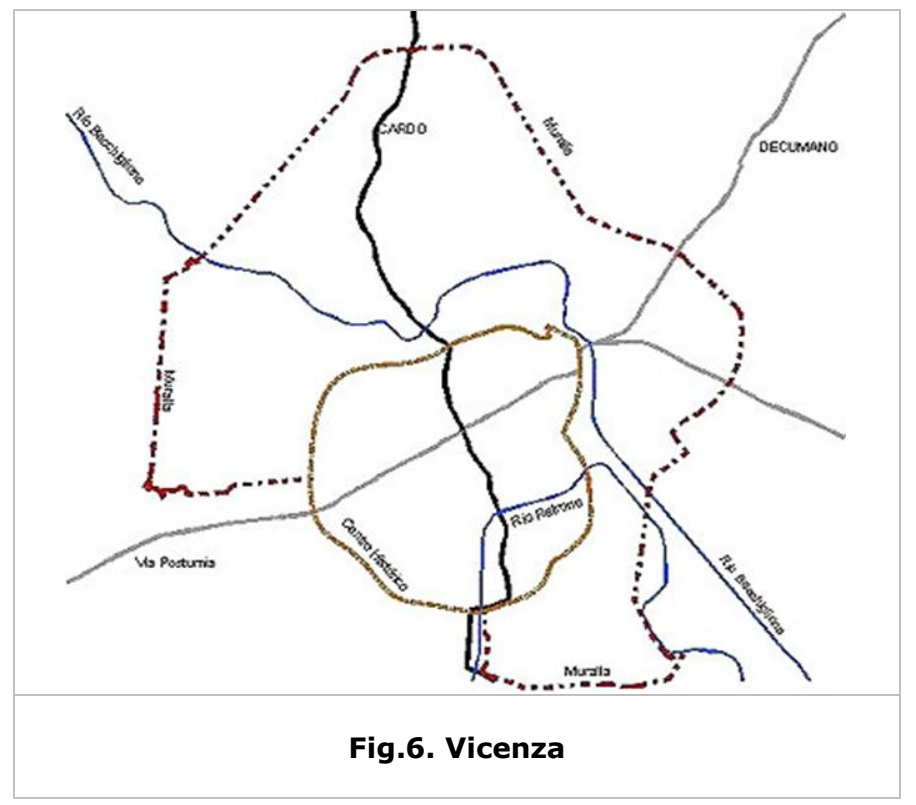

También en Vicenza se difundió la cultura técnico-ingenieril, lo que indujo a adecuar el ordenamiento y la organización funcional del territorio urbano bajo parámetros principalmente higienistas. Entre los proyectos de excepcional importancia se encuentran la construcción del trazado ferroviario y el desplazamiento de la confluencia de los ríos Bacchiglione y Retrone.

Estas dos acciones llevaron al replanteamiento y recualificación del sistema de plazas centrales, demoliendo por ello las cárceles existentes, ampliando la sede de los tribunales y demoliendo algunas porciones de pórticos y puertas, como por ejemplo la puerta Lupia, para hacer posibles algunas rectificaciones viales.

En 1845 se inaugura el tramo del ferrocarril que, dentro de la línea Milán-Venecia, relacionaba Vicenza, Verona y Padova. A diferencia de lo que había sucedido en otras ciudades del Véneto, en Vicenza la creación de una nueva "Puerta Urbana" representada por la estación ferroviaria, no generó una conexión inmediata entre la ferrovía y la ciudad, de hecho el tejido edificado siguió la propia expansión a lo largo de la directriz orienteponiente, paralela al sistema de colinas, dilatando siempre más los límites de la ciudad.

En 1867, durante el período post-unitario, la primera obra ejecutada por la Administración en materia urbanística fue la conservación de la totalidad del Campo Marzo, área verde a los pies del Monte Bérico, destinándolo a la creación de un gran parque público.

Pocos años más tarde, en 1873, se realiza la conexión entre la ciudad y la estación, la cual se lleva a cabo a través de la apertura de una vía perpendicular, que hasta el día de hoy atraviesa el Campo Marzo. 
A partir de esta acción, fuertemente condicionada por estrategias infraestructurales de carácter nacional, el nuevo ordenamiento urbano vicentino se caracterizaba por el desplazamiento del polo de desarrollo, tanto residencial como industrial hacia el sector poniente, mientras el trazado de la ferrovía había determinado la fractura definitiva con el sistema de colinas.

Entre fines de 1870 y los inicios de 1880 se realiza el desplazamiento de la confluencia de los dos ríos principales, anteriormente mencionados. Esta intervención pretendía un doble objetivo: por una parte se buscaba resolver el antiguo problema de las inundaciones que dañaban frecuentemente a esta zona, por otra, el direccionamiento de un programa de transformación del sector de "la Isla" de modo de otorgar a este núcleo una forma urbana completamente renovada.

Las dos intervenciones citadas muestran cómo algunas temáticas de la ingeniería urbanística más fuertemente discutidas en aquellos años -higiene y salubridad de un lado, modernización y extensión del sistema de servicios, del otro-, se convertían, también para Vicenza, en objetivo de grandes reflexiones en torno a la definición de un programa para la ciudad que veía como uno de los objetivos principales solucionar el problema de las inundaciones. A partir de esto ya se estará en condiciones de encargarse de la dotación de aquellos instrumentos esenciales para la gestión de la expansión del territorio urbano: las redes de servicios. Para la Administración se trataba de dar respuesta a una serie de exigencias básicas como la realización del acueducto, del sistema de suministro de energía eléctrica, el potenciamiento de la red de distribución de gas y, muchos años más tarde, la construcción del alcantarillado general.

Profundizando en la temática relativa a los servicios, también importante fue la solución del problema ligado a la conexión del núcleo central con los barrios periféricos. La primera tentativa se inauguró en 1884 a partir de un sistema de coches de tracción animal, el cual, tras unos meses de funcionamiento, fracasó debido a los altos costos para la empresa concesionaria. Casi veinte años más tarde, el Concejo Comunal vuelve sobre el tema del transporte público y en 1909 la Hacienda Municipal asume directamente el servicio, realizando esta vez una línea de tranvía eléctrico que vinculaba el Borgo Pádova con la estación ferroviaria.

A la progresiva realización de la infraestructura sucedió, como era previsible, un decisivo proceso de aceleración de la actividad edificatoria. La rápida sustracción de áreas libres al centro de la ciudad y de su periferia había comenzado en torno al año 1850, ya en 1880 estaba terminándose la primera circunvalación externa al núcleo urbano, a partir de la ejecución de las avenidas Milano y Margherita.

Hasta ese momento se habían conservado al interior del área urbana, amplias zonas de cultivo denominadas piardas. En los primeros años del siglo XX la idea de que estas áreas podrían ser de interés para los planes reguladores de ampliación, adquiría un mayor consenso, de esta forma se lograría densificar el tejido edificado y, en alguna medida, se resolvería la problemática de la necesidad de habitación. 


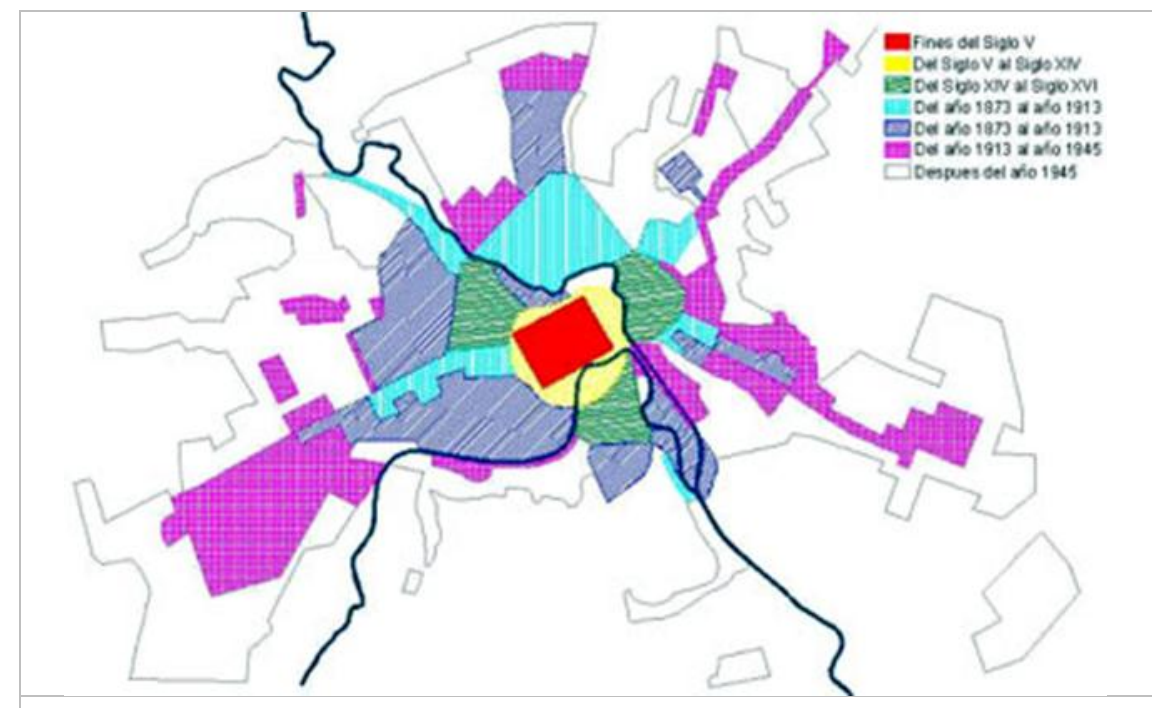

Fig.7. Crecimiento de Vicenza a través de los siglos.

Sobre el plano demográfico, el siguiente período se caracterizó por un fuerte incremento de población, cuyo origen se debía a la difusión en la ciudad de numerosas e importantes actividades industriales con el consiguiente desplazamiento de la mano de obra del campo, constituida en su mayor parte de una población muy pobre, empujada en la búsqueda de mayor bienestar.

Estos movimientos migratorios contribuían a poner de manifiesto la fuerte tensión por la habitación, ya verificada a través de una detallada encuesta dirigida por la Junta Municipal a partir de 1874. De esta indagación emergía una situación de grave precariedad que, a pesar de las intervenciones sucesivas, sería dilatada hasta fines de los años 1930.

En el primer decenio del siglo XX, Vicenza, siguiendo las orientaciones dictadas por las nuevas soluciones legislativas en materia de municipalización de los servicios públicos (ley, 29 marzo 1903, N¹03) y de edificación económica y popular (ley del 31 de mayo 1903, $N^{\circ} 254$, más conocida como Ley Luzzati), se empeñaba en la búsqueda de soluciones apropiadas para los urgentes problemas que la aquejaban y en particular para aquellos relacionados al más reciente fenómeno del urbanismo. Así, en 1906, tres años después de la emanación de la Ley Luzzati, siguió una serie de interrogantes sobre la necesidad de intervenciones para resolver "las desastrosas condiciones de los alojamientos populares". El Concejo Comunal aprobaba la destinación de recursos que permitieron la adquisición de algunos terrenos en las cercanías del Borgo San Felice y de Contra Forti de San Francesco, donde se preveía realizar los primeros proyectos de viviendas populares. Se llevó a cabo un total de 50 alojamientos en esta primera ocasión. El mayor número de intervenciones en materia de construcción de nuevas unidades habitacionales para población de escasos recursos se concentró en la zona norponiente de la ciudad, debido a la vecindad con las principales actividades industriales.

A fines de los años 1920 se optó por llevar a cabo múltiples programas de resaneamiento de los barrios higiénicamente más degradados, dejando de lado las acciones sobre el tejido histórico central. 


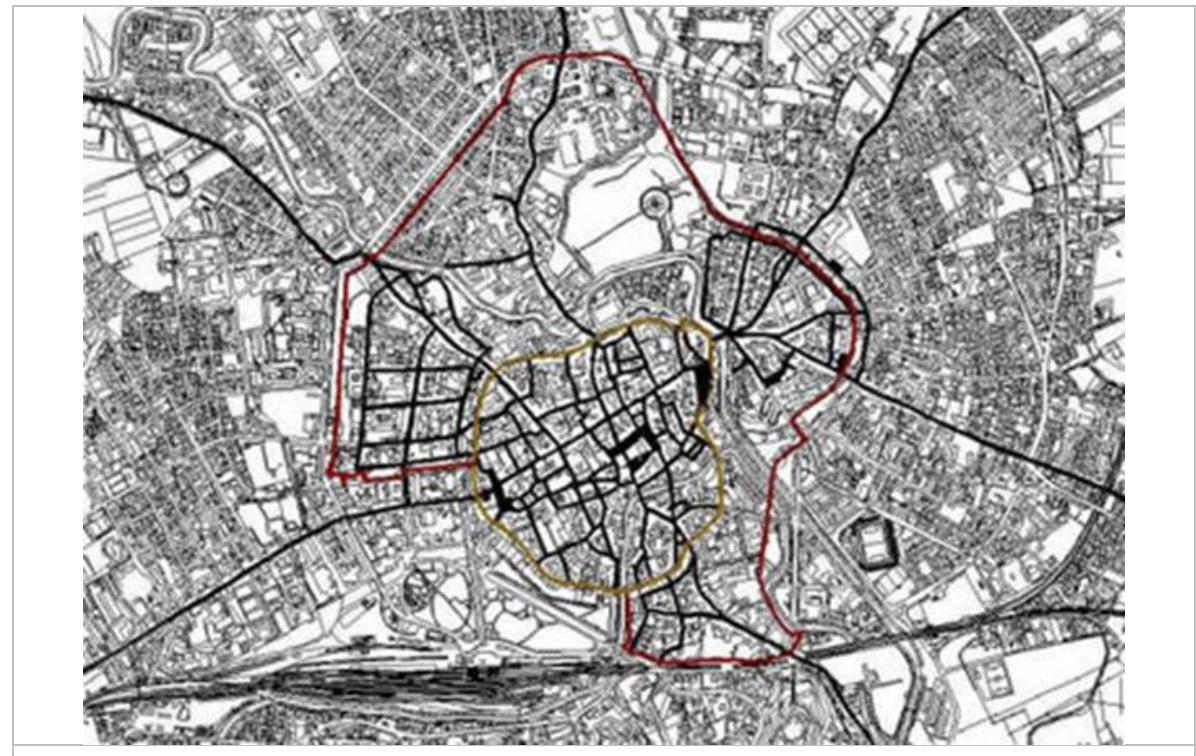

Fig.8. Vicenza

\section{Antecedentes normativos}

Respecto a los instrumentos normativos más significativos que han regido a la ciudad de Vicenza, cabe destacar que en 1876 existía ya un Reglamento de Ornato Público para la ciudad. Dicho reglamento fue sometido a numerosas modificaciones a lo largo del tiempo, es así que en 1895 se aprueba un Nuevo Reglamento de Edificación y Ornato, corregido posteriormente en 1926 y más tarde en 1936, y se incorporan a este reglamento las normas para la protección antiaérea.

También existieron planificaciones parciales que regulaban las condiciones del trazado de las vías para sectores específicos de la ciudad y que eran propuestas por los mismos propietarios de los terrenos edificables.

En 1925 se toma conciencia de la necesidad de realizar un estudio para generar un Plan Regulador General, pero recién en 1932 se nombra a una comisión dedicada al estudio de una propuesta. Años más tarde, entre 1938 y 1939, se lleva a cabo un concurso nacional que busca definir este plan, pero los efectos de la Segunda Guerra Mundial llevaron a la necesidad urgente de crear en primer lugar un Plan de Reconstrucción que entró en vigor en el año 1949. No será sino hasta 1959 que Vicenza contará con un Plan Regulador General.

En 1963 se inician los estudios de Mario Coppa para la generación del Plano Particularizado del Centro Histórico (P.P.C.S.), el cual se profundizará más adelante, y en 1966 se aprueba un Plan para la Edificación Económica Popular (P.E.E.P.). En 1973 se instituye la Oficina del Centro Histórico. 


\section{El plano seccional del centro histórico}

El estudio de Coppa pretendía analizar dos situaciones, por una parte observar y medir los grados de deterioro del centro y por otra, evaluar las potentes dinámicas del distrito y de la provincia vicentina. Los objetivos prefijados eran la descongestión y la recuperación de la residencia, sin embargo, de acuerdo a lo señalado por Gianfranco Franz, el plan no alcanzó a generar soluciones a estos problemas.

"En estas condiciones, el Centro Histórico de Vicenza está condenado: extraída y centrifugada su población, oficinas y actividades administrativas diurnas representan el único sostén; el resultado, si no se pone en marcha un reequilibrio general, será sentido por los vicentinos en los próximos decenios" ${ }^{\prime 2}$.

Coppa además pone en evidencia los descalces entre lo previsto por el Plan Regulador General respecto de las densidades y al número de nuevos habitantes respecto de los números reales que se estaban generando, dejando en claro el despoblamiento del centro. Por ejemplo, en la década del 51 a 61, Vicenza pasó de 58.394 a 75.442 habitantes. En 1951, había 27.908 habitantes en el Centro Histórico y 30.486 en las áreas perimetrales, en cambio en 1961, 24.423 personas $(-12,5 \%)$ habitaban el núcleo y 51.019 (+40\%) la periferia.

"A un Centro Histórico que en osmosis cotidiana acoge y repele a los habitantes, con una periferia de loteos anónimos que poco tienen de urbanidad, se debiera sustituir una dirección de clara programación de las actividades administrativas, llevándolas al exterior, $y$ de recuperación de otras actividades dentro del perímetro del centro histórico y (...) crear las premisas para el retorno a un asentamiento concentrado en torno a los valores tradicionales de las 27.000 - 30.000 unidades $^{\prime \prime}$.

En el año 1969 se vislumbra como solución enfocar la oferta del centro a parejas jóvenes. Para esto se requería resolver en forma prioritaria el tema del tráfico y, en la búsqueda de esta solución, se generaron múltiples proyectos, tales como la reconstrucción del Teatro de la ciudad que había sido destruido por los bombardeos, la generación de una sede universitaria, nuevas conexiones viales, la creación de estacionamientos en torno al perímetro del centro histórico, entre otros, de los cuales muy pocos fueron llevados a cabo en los plazos planificados.

\section{Problemática actual}

A lo largo del tiempo, la actividad económica de Vicenza y sus alrededores ha ido mudando radicalmente, es así que de ser una zona principalmente agrícola se ha ido convirtiendo en un fuerte centro industrial y de actividades terciarias.

Esta transformación ha provocado la llegada de gran cantidad de población extranjera. Dentro de la provincia de Vicenza son más de 34.000 habitantes los de origen extranjero,

2 LEDER, Francesca, SACCARDO, Umberto; VICENZA, Ottocento e Novecento: piani, progetti e modificazioni, Ergon Edizioni, Vivenza 1996, pág 71.

3 LEDER, Francesca, SACCARDO, Umberto. VICENZA, Ottocento e Novecento: piani, progetti e modificazioni, Ergon Edizioni, Vivenza 1996, pág 72. 
de los cuales, cerca de unos 8.000 provienen de la ex Yugoslavia, 5.000 de Marruecos, 3.700 de Ghana y casi 3.000 de Albania. Últimamente se ha incorporado un gran volumen de población de la India, Senegal (grupo conformado en su mayoría de hombres) y Rumania. La comuna de Vicenza es la que presenta el mayor número de habitantes extranjeros residentes con más de 6.000 inmigrantes registrados.

Otro dato que interesa es la transformación que ha sufrido la composición de la familia, en donde el número de integrantes ha disminuido considerablemente; así, tenemos que en el año 1961 cada núcleo familiar estaba compuesto por una media de 4 personas, en cambio el censo del año 2001 nos revela que este número ha decrecido en 2,6 personas por núcleo familiar. Esta variación ha incidido directamente sobre el número de las viviendas existentes, las cuales han aumentado a más del doble: de 160.000 en 1961, tenemos en el 2001 existen un total de 350.000 viviendas $^{4}$.

Pese a esta gran demanda de habitación el Centro Histórico continúa siendo un espacio poco habitado donde, después de la jornada laboral, las calles aparecen prácticamente desiertas.

Los motivos, según lo percibido en nuestra estada, son los altos costos de arriendo de los departamentos en oferta y una carencia de actividades complementarias a la vivienda. Como ya lo señalaba Coppa en su estudio para el plano seccional citado, la presencia de nuevos polos culturales y educacionales traería nueva sangre a este centro.

\section{Red de cooperación urb-al}

URB-AL es un programa horizontal de cooperación descentralizada de la Comisión Europea dirigido al conjunto de ciudades, aglomeraciones y regiones de la Unión Europea y de América Latina, el cual se apoya en redes constituidas sobre una base temática, encaminadas a solucionar problemas concretos de desarrollo urbano local. Las redes temáticas tienen por objeto permitir el intercambio de experiencias, la definición de problemas comunes, la articulación de mecanismos e instrumentos de acción, la difusión de buenas prácticas y el seguimiento de los proyectos comunes seleccionados.

Estos intercambios permiten a los actores locales desarrollar sus capacidades para hacer frente a las exigencias planteadas por el fenómeno de urbanización acelerada que se ha registrado en el transcurso de las últimas décadas.

Dentro de este marco, actualmente se desarrolla el proyecto "Centro internacional de formación para la valorización y conservación de los contextos histórico urbanos", el cual busca reforzar la capacidad de acción de las colectividades locales en este campo, llevando a cabo dos tipos de formación; una, destinada a jóvenes profesionales que tuviesen un lazo directo con las entidades locales implicadas en la red, tratando en particular el concepto de la restauración urbana integrada; otra, destinada a los funcionarios públicos o agentes municipales encargados de la gestión de esta problemática.

Los socios de esta red temática son: El Ayuntamiento de Málaga (España), Consejo Estatal para la Cultura y las Artes de Hidalgo y la Presidencia Municipal de Pachuca de Soto (México), Prefectura de Belem (Brasil), Alcaldía de Villa de Leyva (Colombia), Alcaldía de

4 Fuente: Instituto de Estadística de Italia, ISTAT. 
Nueva San Salvador (El Salvador), Municipalidad de Cuenca (Ecuador), Gobierno Regional de Tarapacá y la Ilustre Municipalidad de Santiago (Chile) y la Provincia de Vicenza (Italia), ésta última como organizadora y sede de los estudios impartidos.

Como participantes del curso de jóvenes profesionales, representando a la Ilustre Municipalidad de Santiago de Chile, nos ha tocado experimentar esta enriquecedora experiencia, asistiendo a tres módulos de clases en la ciudad de Vicenza, en los cuales no sólo adquirimos conocimientos teóricos y prácticos relacionados con las temáticas tratadas en el curso, sino además, a partir de las vivencias durante casi ocho meses, percibimos la problemática asociada a un centro histórico de gran antigüedad y riqueza arquitectónica como es el de Vicenza.

Dentro del módulo práctico del curso, se nos encargó analizar el barrio de San Rocco, el cual antiguamente se conocía como barrio de Porta Nuova; barrio que siendo parte del Centro Histórico aparece desvinculado de éste, con un alto nivel de degrado tanto en sus edificaciones como en sus espacios de circulación.

El encargo específico era elaborar un proyecto de rehabilitación del Claustro de Santa María Nova. Puesto que, de acuerdo a lo expuesto por el profesor Vincenzo Zucchi, profesor de Urbanismo del Politécnico de Milán, la rehabilitación de un área no se logra con el sólo hecho de generar un elaborado plan especial, con normativas que apunten a su consolidación futura y que sea fruto de un análisis detallado de sus tipologías edilicias. Esto constituye una gran base sobre la cual trabajar, pero debe ser apoyada e impulsada a partir de la generación de "proyectos detonantes", que aumenten la calidad de vida de los residentes, incentiven el mejoramiento de los espacios públicos y por consiguiente atraiga el interés de una nueva población a instalarse en él.

El proyecto requería realmente una yuxtaposición de acciones, en donde, de acuerdo a lo aprendido, debíamos aplicar los conceptos de restauración del edificio original, demolición de todos los agregados que constituían falsos arquitectónicos, es decir que simulaban ser de la época original pero no lo eran. Incorporando también el concepto de reciclaje, puesto que este antiguo claustro debía convertirse en un Centro Internacional de Formación para la Valorización y la Conservación de Contextos Históricos Urbanos, para lo cual muchos espacios debían ser adaptados a los nuevos usos. Finalmente, se requería de la construcción de obra nueva, en donde se alojaron la biblioteca, los talleres y las salas orientadas a la aplicación de nuevas tecnologías.

El Centro de estudios, elaborado en un conjunto por cinco estudiantes, se concibió como un centro abierto, en donde los vecinos estarían invitados a participar de las actividades tanto deportivas como culturales, utilizando su gimnasio, la biblioteca y su Sala de Conferencias, además de generar nuevos espacios públicos, que hoy en día son casi inexistentes en este barrio. 


\section{Vicenza, el nuevo desarrollo urbano a partir del Programa de Recalificación Urbana y del Desarrollo Sostenible del Territorio, P.R.U.S.S.T}

En noviembre de 2003 se realizó en Cannes la feria mundial de urbanismo y de mercado inmobiliario. Vicenza como una de las ciudades arquitectónicamente más interesantes de Italia, se mostró muy interesada en su proyección hacia el futuro y enfocada hacia el ámbito de afrontar dimensiones europeas y mundiales.

"La administración comunal de Vicenza,- explica el alcalde de la ciudad Enrico Hüllweck, - ha puesto como centro de su actividad el desarrollo urbano y la recalificación del territorio. El objetivo principal de esta acción es garantizar a nuestra ciudad un centro moderno, funcional, promoviendo, al mismo tiempo, la calidad de vida y la vigilancia del ambiente".

El Departamento territorial de la comuna de Vicenza ha coordinado un complejo programa para el desarrollo económico de la ciudad, a partir de la realización de 27 proyectos. Con tal programa la ciudad estaría dotada de funciones urbanas excelentes, entre los cuales están el nuevo teatro ciudadano, el espacio de la justicia, un polo museístico, la universidad, un polo bibliotecario y un barrio para exposiciones y ferias.

Tal programación se realiza a través del Programa de Recalificación Urbana y del Desarrollo Sostenible del Territorio, P.R.U.S.S.T. Gran tarea asume el programa, la serie de intervenciones para la revitalización del centro histórico, la recuperación y la refuncionalización del área y la restauración conservativa de ilustres monumentos de la ciudad, tales como, la Basílica Palladiana y el conjunto Santa Corona.

Por otra parte, se contempla el desarrollo de la infraestructura que mejore el sistema de transporte público, la realización de un sistema de intercambio modal, una red de recorridos peatonales y ciclovías y la generación de nuevos estacionamientos.

Por cuanto resguarda la actividad comercial, la administración comunal ha adecuado la programación de las instalaciones comerciales sobre la base de las direcciones y criterio regional. La ciudad estará dotada de servicios para el desenvolvimiento de actividades promocionales para el artesano y la industria local, valorizando el centro histórico a través de numerosas manifestaciones que hacen vivir la tradición, los usos y costumbres y los productos locales. La feria de Vicenza representa un importante polo de atracción de nivel internacional famosa en todo el mundo para el sector del oro. Intervenciones programadas en la feria intentan desarrollarse y reorganizarse al interior del barrio donde se emplaza la feria. El sistema cultural representa el componente más importante al determinar la identidad de una comunidad y de un asentamiento urbano. La realización del nuevo teatro ciudadano representa un polo capaz de hacer de catalizador y propulsor de la programación artística ciudadana y nacional.

El sistema de recuperación del patrimonio histórico, arquitectónico y ambiental es un tema fundamental dentro de una política con miras a la realización de una calidad urbana sustentable. 
Vicenza posee un patrimonio histórico arquitectónico de notable valor que necesita de importantes intervenciones de restauración, y de los espacios verdes excepcionales que en el curso de los últimos decenios, han perdido sus características más importantes.

Las 27 intervenciones previstas por el P.R.U.S.S.T., han activado recursos públicos y privados. El cronograma definitivo con la suscripción del acuerdo entre el Ministerio de Infraestructura y Transporte, la Región del Véneto, la Comuna de Vicenza (agente promotor) y el agente proponente, prevé que la actuación del programa se lleve a cabo en un plazo de diez años.

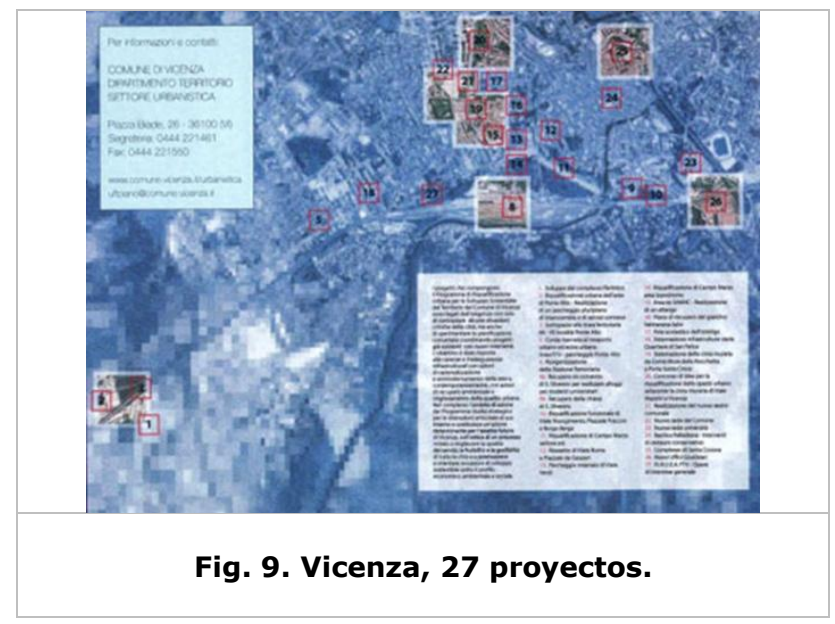

\section{Bibliografía}

LEDER, Francesca; SACCARDO, Umberto. VICENZA, Ottocento e Novecento: piani, progetti e modificazioni, Ergon Edizioni, Vivenza 1996.

REVISTA SET; número 26 / 2003, Vicenza il nuovo svilupo urbano.

COMUNE DI VICENZA - Dipartimenti Ilpp e Territorio, Settori Progettazioni ed Urbanistica; Strudio di fattibilita' per il recupero, il restauro e la valorizzazione delle mura de Vicenza.

http://urb-al.provincia.vicenza.it

http://urb-al.provincia.vicenza.it

http://www.cisapalladio.org 